\title{
INTERVENÇÃO FISIOTERAPÊUTICA PRECOCE EM LACTENTES DE RISCO: UMA VIVÊNCIA BASEADA EM EVIDÊNCIAS
}

\author{
Carine Gomes De Fáveri \\ Universidade Federal do Triângulo Mineiro \\ carinegdefaveri@gmail.com \\ Roberta Jéssica Silva Pires \\ Universidade Federal do Triângulo Mineiro \\ robertajpires@gmail.com \\ Isabella Polo Monteiro \\ Universidade Federal do Triângulo Mineiro \\ isabellapm10@gmail.com
}

\author{
Marília Carvalho Borges \\ Universidade Federal do Triângulo Mineiro \\ maa.borgess19@gmail.com
}

Mariana Aparecida de Assis Campos Universidade Federal do Triângulo Mineiro mcamposfisioterapeuta@gmail.com

Elaine Leonezi Guimarães Universidade Federal do Triângulo Mineiro elaine.guimaraes@uftm.edu.br

\section{Resumo}

Objetivo: Descrever evidências práticas da intervenção fisioterapêutica, vivenciadas no projeto de extensão "Intervenção precoce para lactentes de risco", e a contribuição deste na formação profissional de acadêmicos extensionistas e residentes. Método: De 2014 a 2019, foram atendidos 380 lactentes, sendo selecionados para o estudo 307 prontuários com dados completos sobre avaliação, intervenção e reavaliação fisioterapêutica dos lactentes. Resultados: Todos os lactentes considerados de risco devido à prematuridade e/ou baixo peso ao nascimento, apresentaram alterações no desenvolvimento sensório-motor, os quais foram e/ou continuam sendo acompanhados até completarem dois anos. Conclusão: Os resultados permitiram discutir evidências referentes às condições de risco pré, peri e pós-nascimento, a importância da detecção e intervenção precoces no desenvolvimento do lactente de risco, e a participação da família neste processo. Além disso, o projeto permitiu capacitar melhor os acadêmicos e residentes na área de atenção à saúde da criança, favorecendo o cuidado humanizado do lactente na sua integralidade.

Palavras-chave: Lactente de Risco. Intervenção Precoce. Fisioterapia.

\section{EARLY PHYSIOTHERAPEUTIC INTERVENTION IN RISK INFANTS: AN EVIDENCE-BASED EXPERIENCE}

\section{Abstract}

Objective: To describe practical evidence of physical therapy intervention, experienced in the extension project "Early intervention for high-risk infants", and its contribution to the professional training of extension students and residents. Method: From 2014 to 2019, 380 infants were treated, with 307 records selected for the study, with complete data on the assessment, intervention and physiotherapeutic reevaluation of infants. Results: All infants considered at risk due to prematurity and/or low birth weight, presented changes in motor sensory development, which were continue to be followed up until they are two years old. Conclusion: The results made it possible to discuss evidence regarding the pre, peri and post-birth conditions, the importance of early detection and intervention in the development of the at-risk infant, and the family's participation in this process. In addition, the project allowed for better training of academics and residents in the area of child health care, favoring the humanized care of infants in their entirety.

Keywords: Infant at Risk. Early Intervention. Physiotherapy.

\section{INTERVENCIÓN FISIOTERAPÉUTICA TEMPRANA EN LACTANTES DE RIESGO: EXPERIENCIA BASADA EN EVIDENCIA}

\section{Resumen}

Objetivo: Describir la evidencia práctica de la intervención de fisioterapia, experimentada en el proyecto de extensión "Intervención temprana para lactantes de alto riesgo", y su contribución a la formación profesional de estudiantes de extensión y residentes. Método: De 2014 a 2019 se trataron 380 lactantes, con 307 registros seleccionados para el estudio, con datos completos sobre la evaluación, intervención y reevaluación fisioterapéutica de los lactantes. Resultados: Todos los lactantes considerados de riesgo por prematuridad y/o bajo peso al nacer, presentaron cambios en el desarrollo sensorial motor, los cuales fueron y continúan en seguimiento hasta los dos años. Conclusión: Los resultados permitieron discutir evidencias sobre las condiciones de riesgo pre, peri y posnatal, la importancia de la detección e intervención tempranas en el desarrollo de los lactantes de alto riesgo y la participación de la familia en este proceso. Además, el proyecto permitió una mejor formación de académicos y residentes en el área de la salud infantil, favoreciendo la atención humanizada de la infancia en su totalidad.

Palavras claves: Infante de Riesgo. Intervención Rápida. Fisioterapia. 
Intervenção fisioterapêutica precoce em lactentes de risco: uma vivência baseada em evidências

\section{INTRODUÇÃO}

A intervenção precoce é aquela realizada antes que se detectem anormalidades no desenvolvimento do lactente, ou seja, antes que as manifestações clínicas próprias da doença estejam evidentes, como: alteração do tônus postural, da postura, dos movimentos e das coordenações sensório-motoras primárias, comprometendo a função do bebê (CABRAL et al., 2016; FORMIGA; PEDRAZZANI; TUDELLA, 2010).

Os lactentes elegíveis para receber tal intervenção são aqueles considerados de risco, devido a determinantes biológicos e ambientais, necessitando de internação em Unidades de Terapia Intensiva Neonatal e acompanhamento ambulatorial (FORMIGA; LINHARES, 2009).

Há evidências de que quanto mais precoce for o diagnóstico e a intervenção nas possíveis alterações clínicas do lactente considerado de risco, menor será o impacto dessas alterações na aquisição da funcionalidade e, consequentemente, no desenvolvimento neurossensoriomotor sendo essencial para o sucesso da intervenção, a adesão e participação dos pais ou cuidadores (FORMIGA; RAMOS, 2016; VALVERDE; JURDI, 2020).

Diversos fatores podem refletir negativamente no desenvolvimento da criança, sendo denominados fatores de risco. Estes podem ser: a prematuridade (idade gestacional menor que 37 semanas), baixo peso ao nascimento (menor que 2500 gramas), exposição a agentes infecciosos, lesões, desigualdade social, racismo, falta de oportunidades, pobreza, entre outros, que se não forem tratados imediatamente, podem evoluir e tornar-se problemas mais difíceis de serem resolvidos (SANTOS; PACHECO, 2016). Desta forma, é indicado o acompanhamento dessas crianças para que sejam identificadas as possíveis condições e/ou sinais de risco, e iniciada a intervenção precoce, a fim de favorecer o desenvolvimento das mesmas (SANTOS; PORTO; LERNER, 2014).

Considerando o desenvolvimento motor um processo contínuo de amadurecimento físico, psíquico e social, relacionado à plasticidade neural e maturação cerebral contínua, durante a primeira infância (MARINI; LOURENÇO; BARBA, 2017), é necessário que se priorize programas de assistência longitudinal (Follow up), com o intuito de estabelecer estratégias eficazes para a intervenção, bem como, para prevenir possíveis alterações neurossensoriomotoras (FORMIGA; PEDRAZZANI; TUDELLA, 2010; NOVAK; MORGAN, 2019).

Pensando nisso, e detectado a escassez de serviço especializado no acompanhamento do desenvolvimento motor do lactente de risco na cidade, foi idealizado o projeto de extensão

Extensio: R. Eletr. de Extensão, ISSN 1807-0221 Florianópolis, v. 18, n. 39, p. 25-35, 2021. 
Intervenção fisioterapêutica precoce em lactentes de risco: uma vivência baseada em evidências

"Intervenção Precoce para Lactentes (IPL)", com a criação de um ambulatório, junto ao Serviço de Pediatria do Hospital de Clínicas da Universidade Federal do Triângulo Mineiro (UFTM/EBSERH), para avaliar e acompanhar o lactente de risco nos dois primeiros anos de idade. O projeto de extensão tem por objetivos: acompanhar o desenvolvimento dos lactentes de risco durante os dois primeiros anos de idade; detectar possíveis sinais indicativos de alterações no desenvolvimento motor; estimular as crianças e/ou encaminhar para outros serviços, quando necessário; orientar pais e responsáveis na estimulação domiciliar; observar e descrever o efeito da fisioterapia motora precoce; e, ainda, contribuir para a formação de acadêmicos do curso de Fisioterapia e residentes fisioterapeutas na área de atenção à saúde da criança.

Assim, o objetivo do presente artigo foi descrever evidências práticas da intervenção fisioterapêutica vivenciadas no projeto de extensão, por meio de resultados alcançados junto aos lactentes de risco, bem como, a contribuição deste na formação profissional dos acadêmicos extensionistas e residentes.

\section{MATERIAIS E MÉTODOS}

O presente estudo trata-se de um relato de experiência, do tipo documental, retrospectivo, de caráter analítico, descritivo, baseado nas ações desenvolvidas no projeto de extensão "Intervenção Precoce para Lactentes (IPL)", junto ao Ambulatório de Pediatria do Hospital de Clínicas da FTM/EBSERH, cujos atendimentos são realizados duas vezes na semana.

Os lactentes são encaminhados por médicos pediatra e neonatologista, e têm o consentimento livre e esclarecido dos pais ou responsáveis para os atendimentos. Os lactentes são avaliados e acompanhados durantes os dois primeiros anos de vida, ou até adquirir a marcha independente. Entretanto, para o presente estudo, considerando que para ser precoce a intervenção deve-se iniciar antes dos seis meses de idade, foram apresentados e discutidos os dados dos lactentes nesta faixa etária.

Os dados analisados no presente estudo são parte dos dados coletados no projeto de Pesquisa "Análise das categorias indicativas de risco para alterações no desenvolvimento de lactentes, segundo o instrumento Avaliação do Desenvolvimento Neurossensoriomotor do Bebê de Risco", aprovado pelo Comitê de Ética em Pesquisa (CEP) da Universidade Federal do Triângulo Mineiro - UFTM, parecer n 2115516.

Extensio: R. Eletr. de Extensão, ISSN 1807-0221 Florianópolis, v. 18, n. 39, p. 25-35, 2021. 
Intervenção fisioterapêutica precoce em lactentes de risco: uma vivência baseada em evidências

No período de 2014 a 2019, foram atendidos 380 lactentes no projeto, e capacitados 30 extensionistas acadêmicos do curso de Fisioterapia e 6 residentes (fisioterapeutas) em atenção à saúde da criança. Para o presente estudo foram selecionados 307 prontuários, os quais apresentavam dados completos de internação, da avaliação antes de iniciar a fisioterapia e reavaliações durante o período de acompanhamento e intervenção. O instrumento utilizado para avaliação dos lactentes de zero a seis meses no projeto foi "Avaliação do Desenvolvimento Neurossensoriomotor do Bebê de Risco" (GUIMARAES, 2001). O instrumento permite avaliar cinco categorias, sendo elas: tônus muscular (TM); posturas: supino (PS), prono (PP), tracionado para sentado (TS) e sentado com apoio (S); reflexos primitivos (RP); reações posturais (RPos); e coordenações sensório-motoras primárias (CSMP), dos 20 aos 180 dias de vida pós-natal, com o objetivo de identificar possíveis riscos até os seis meses de idade. A pontuação total obtida na avaliação, sendo diferente de zero, sugere risco para possível atraso no desenvolvimento sensório-motor, indicando a necessidade de acompanhamento e intervenção precoce.

Todos os lactentes que apresentaram sinais clínicos indicativos de risco ao nascimento (prematuridade, baixo peso ao nascer, hipóxia neonatal, síndromes, lesões do sistema nervoso central e periférico e ortopédicas), e, alterações na avaliação do desenvolvimento motor, foram submetidos à fisioterapia motora uma ou duas vezes na semana. A intervenção era feita por meio de técnicas baseadas no neurodesenvolvimento e sistemas dinâmicos, em salas com divãs, colchonetes, brinquedos coloridos e sonoros de diversos tamanhos e texturas, sempre com a presença dos pais ou cuidadores. A duração média das sessões era de 30 a 40 minutos.

Para orientação dos pais e cuidadores, foram elaborados e entregues durante as sessões folhetos informativos sobre o desenvolvimento motor, cuidados com o lactente de risco, e atividades indicadas para estimulação domiciliar.

Os extensionistas (acadêmicos e residentes), participaram das atividades práticas (avaliação, intervenção, evolução e orientações), e, de atividades didático-pedagógicas (leitura e discussão de artigos, apresentação de casos, elaboração e apresentação de trabalhos em eventos científicos e extensionistas).

Os dados coletados das avaliações foram plotados no Software SPSS (Statistical Package for Social Sciences) versão 20.0, para posterior análise e caracterização dos lactentes, de acordo com os fatores de risco. Para o tratamento estatístico dos dados, considerando a variabilidade da população de lactentes, foi utilizada estatística descritiva simples, por meio de frequência relativa e absoluta, média e desvio padrão.

Extensio: R. Eletr. de Extensão, ISSN 1807-0221 Florianópolis, v. 18, n. 39, p. 25-35, 2021. 
Intervenção fisioterapêutica precoce em lactentes de risco: uma vivência baseada em evidências

A análise descritiva do impacto do projeto na formação profissional de acadêmicos e residentes foi baseada nos relatórios finais apresentados à Pró-Reitora de Extensão durante as seis edições do projeto, no período de 2014 a 2019.

\section{RESULTADOS}

Quanto à caracterização dos lactentes, observou-se que 169 (33,3\%) eram meninos e 138 $(27,2 \%)$ meninas. A idade gestacional média foi de 34,6 $( \pm 3,95)$ semanas e, peso médio ao nascimento, de 2181,4 ( $\pm 832,2)$ gramas. Verificou-se que 173 (56,4\%) foram pré-termo, 203 (66,1\%) tiveram peso menor que 2.500 gramas, 15 (4,9\%) com apresentação pélvica, e 24 (7,8\%) gemelares.

Como fatores pré-natais, observou-se o perfil das mães, verificando-se média de idade de $27,7( \pm 7,37)$ anos; 51 (16,6\%) foi mãe antes dos 20 anos; 47 (15,4\%) mãe após os 35 anos; 209 (68\%) tiveram o primeiro parto entre 20 e 35 anos de idade. Observou-se ainda número médio de gestação de $1,9( \pm 1,1)$; de parto prematuro anterior $1,8( \pm 0,38)$; e, histórico de morte fetal anterior em 1,8 $( \pm 0,38)$ das mães.

$\mathrm{Na}$ análise referente aos dados dos lactentes no período pós-natal, verificou-se que 226 (73,6\%) necessitaram permanecer hospitalizadas por mais de 2 dias. Destas, 207 (67,6\%) ficaram em incubadoras, 89 (29\%) apresentaram icterícia neonatal, 156 (50,8\%) necessitaram de fototerapia, e 92 (30\%) apresentaram insuficiência respiratória. A necessidade de reanimação foi observada em $67(21,9 \%)$ lactentes e 20 (6,5\%) apresentaram hipóxia. Observou-se, ainda, o diagnóstico clínico de malformação congênita em 35 (11,4\%) lactentes e 23 (7,5\%) com síndrome genética.

$\mathrm{Na}$ avaliação fisioterapêutica pós-alta hospitalar, com base nas cinco categorias abordadas pelo instrumento de avaliação, 100\% dos lactentes apresentaram alterações, sendo: $51,15 \%$ em tônus muscular, 52,44\% na postura supina, 59,93\% na postura prona, 57\% na postura tracionado para sentado, 56,02\% na postura sentado com apoio, 43,97\% em reflexos primitivos, 59,28\% em reações posturais, e, 42,02\% em coordenações sensório-motoras primárias.

Os resultados observados por meio da avaliação nos primeiros seis meses de idade indicam que, desde a primeira avaliação, os lactentes apresentavam algum sinal indicativo de risco, com maior pontuação de risco observada nas avaliações aos 60, 90, 120, 150 e 180 dias

Extensio: R. Eletr. de Extensão, ISSN 1807-0221 Florianópolis, v. 18, n. 39, p. 25-35, 2021. 
(Figura 1). Entretanto, aos 30 dias a pontuação já sinalizava risco, porém, poucas crianças foram avaliadas aos 30 dias, seja por ainda estar em internação hospitalar ou não terem sido encaminhadas ao ambulatório.

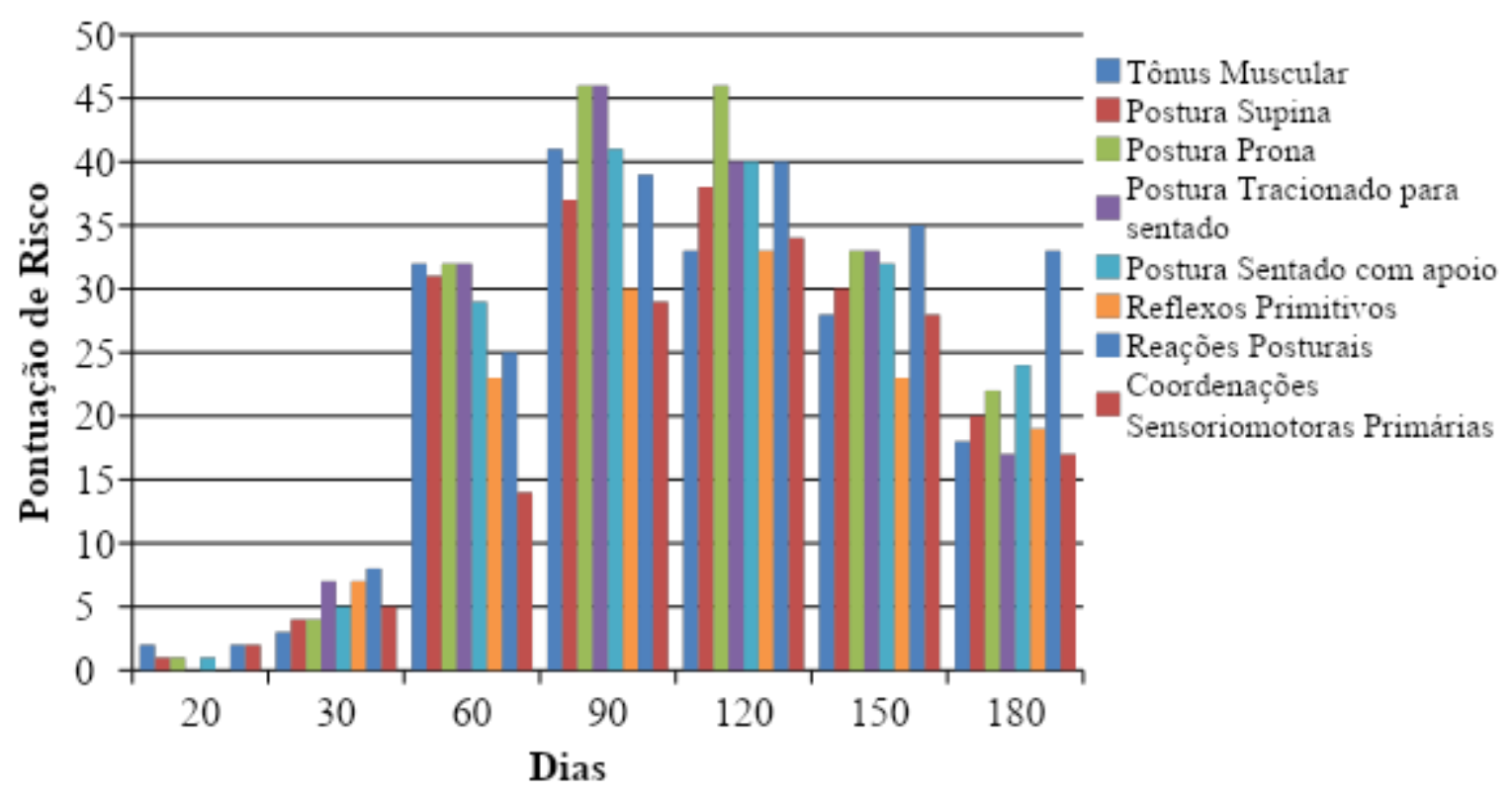

Figura 01 - Pontuação de risco observada nas avaliações entre zero e seis meses de idade cronológica, considerando as categorias (tônus muscular, postura supina, postura prona, postura tracionado para sentado, postura sentado com apoio, reflexos primitivos, reações posturais e coordenações sensório-motoras primárias). Uberaba, MG. 2020. Fonte: elaborado pelas autoras.

Dos 307 lactentes avaliados, 185 (60,3\%) necessitaram de intervenção fisioterapêutica precoce no ambulatório, e, $122(39,7 \%)$ os pais apenas recebiam orientações para estimulação em casa, sendo realizado acompanhamento ambulatorial mensal do desenvolvimento de todas as crianças durante os dois primeiros anos de vida.

Quanto a contribuição do projeto para a formação profissional dos acadêmicos e residentes, verificou-se importante impacto na formação e capacitação destes, de acordo com os relatórios finais das seis edições do projeto de extensão, no período de 2014 a 2019.

O elevado número de atendimentos registrados, o treino para a aplicação das escalas de avaliação, execução dos métodos e técnicas de intervenção específicas, contribuíram para capacitação por meio da prática em serviço, demonstrando impacto positivo do projeto de extensão no ensino. Além disso, diversos trabalhos de pesquisa foram realizados e apresentados em eventos científicos e extensionistas, alcançando o objetivo de indissociabilidade da tríade ensino, pesquisa e extensão. 
Intervenção fisioterapêutica precoce em lactentes de risco: uma vivência baseada em evidências

Vale ressaltar, que novas perspectivas de trabalhos de extensão e pesquisa surgiram no decorrer das edições do projeto. E, ainda, foi possível observar a importância do mesmo, na aprovação dos egressos do projeto, em processos seletivos de programas de residência e mestrado.

\section{DISCUSSÃO}

Considerando o objetivo do presente estudo em descrever evidências práticas da intervenção fisioterapêutica vivenciadas no projeto de extensão, os resultados observados no decorrer do projeto nos permitem discutir evidências referentes às condições pré, peri e pósnatal do lactente de risco, características da mãe, importância do acompanhamento, da detecção e intervenção precoces nos primeiros meses de vida do lactente.

Quanto aos fatores do pré-natal relacionados à mãe, verificou-se que a idade materna menor que 20 e maior que 40 anos foi um fator de risco comum, corroborando com a literatura (ALMEIDA et al., 2018). Considera-se a idade materna ideal para reprodução, de 20 a 29 anos. Gestação muito precoce, ou seja, antes dos 20 anos, é considerada de risco pela imaturidade biológica, e desenvolvimento incompleto da estrutura óssea da mãe, assim como gestações tardias após os 35 anos estão mais propensas às complicações obstétricas (SILVA et al., 2020).

Considerando os fatores de risco perinatais, o baixo peso ao nascimento e a prematuridade são os mais comuns para o recém-nascido. Ambos corroboram para o maior risco de mortalidade neonatal, bem como para o surgimento de sequelas durante o desenvolvimento motor (CHASKEL et al., 2018; SETUMBA et al., 2018). Da população do estudo, 56,4\% dos lactentes nasceram prematuros, $21,9 \%$ necessitaram de reanimação, e, 17,9\% de internação por mais de dois dias, e todos apresentaram sinais indicativos de risco durante as avaliações no pós-natal, corroborando os dados encontrados nos estudos de Savio et al. (2016), Granzotto, Fonseca e Lindemann (2012), e, Krey et al. (2016).

Vale ressaltar que entre os fatores de risco para alterações no desenvolvimento, o baixo peso ao nascimento e a prematuridade foram os mais frequentes no presente estudo. De acordo com a literatura, crianças com baixo peso $(<2.500$ gramas $)$ apresentam déficits no desenvolvimento motor até atingirem 3 anos de idade (WALKER et al., 2007). Ademais, a prematuridade e o baixo peso ao nascimento estão entre as principais causas de atraso no desenvolvimento neuropsicomotor, sendo importante a intervenção precoce para a habilitação

Extensio: R. Eletr. de Extensão, ISSN 1807-0221 Florianópolis, v. 18, n. 39, p. 25-35, 2021. 
Intervenção fisioterapêutica precoce em lactentes de risco: uma vivência baseada em evidências

do lactente de risco, e se torna mais eficaz, quando há a participação dos familiares (SILVA, 2017).

No presente estudo, também foi observado a necessidade de fototerapia devido à presença de icterícia neonatal em 50,8\% dos lactentes, indicando mais uma condição de risco conforme descrito na literatura (SABOUTE et al. 2017). A hiperbilirrubinemia até 36 horas após o nascimento pode causar sequelas neurológicas (MESQUITA; CASARTELLI, 2017), devendo ser tratada o mais precocemente possível.

Considerando os resultados da avaliação do desenvolvimento motor, observou-se alterações nas variáveis tônus muscular, reflexos primitivos, postura, reações posturais, e coordenações sensório-motoras primárias em todos os lactentes, com pontuação diferente de zero e em maior evidência aos 60, 90,120,150 e 180 dias de idade.

As alterações no tônus muscular foram observadas em todas as idades, embora tenderam a diminuir após os 90 dias, corroborando o estudo de Urzêda et al. (2009). Contudo, é um fator importante, pois dificulta o controle nas diversas posturas, altera a inibição dos reflexos primitivos, consequentemente, retarda a aquisição das reações posturais e coordenações sensório-motoras primárias.

Já as alterações nas posturas prona, tracionado para sentar e sentado com apoio, mais evidentes entre 60 a 150 dias, corrobororaram os resultados obtidos por Guimarães (2001), sendo importante a estimulação das mesmas, bem como orientar os pais ou cuidadores a colocar o lactente nas diversas posturas.

Quanto aos resultados referentes às categorias reações posturais e reflexos primitivos, foram observadas alterações mais evidentes entre os 60 e 180 dias. Tais resultados corroboram com os achados do estudo de Guimarães e Tudella (2003), utilizando o mesmo instrumento de avaliação, e, em população com perfil semelhante.

Considerando que na avaliação utilizada durante os primeiros seis meses de idade cronológica, pontuações diferentes de zero é considerado risco para atraso, 100\% dos lactentes apresentaram sinais de risco, por isso foram incluídos no projeto. Assim, com base na análise das condições clínicas e funcionais, os lactentes foram incluídos nos grupos de intervenção fisioterapêutica ou de orientação aos pais para estimulação domiciliar. Vale ressaltar, que todos os lactentes continuaram sendo avaliados após os seis meses de idade, por meio da Escala Motora de Alberta (AIMS) (Piper; Darrah, 1994).

Extensio: R. Eletr. de Extensão, ISSN 1807-0221 Florianópolis, v. 18, n. 39, p. 25-35, 2021. 
Intervenção fisioterapêutica precoce em lactentes de risco: uma vivência baseada em evidências

Importante destacar, ainda, que a população do presente estudo alcançou o desenvolvimento motor esperado até os dois anos de idade de corrigida. Entretanto, se não alcançasse, seria encaminhada para programas de intervenção e estimulação de crianças maiores.

Considerando que mais de 200 milhões de crianças com idade inferior a 5 anos não alcançam seu potencial de desenvolvimento (NutriSUS, 2015), são necessários estudos que identifiquem os fatores de risco aos quais as crianças estão expostas, bem como projetos e programas de acompanhamento e intervenção precoce para lactentes, e, para crianças maiores.

Quanto à contribuição do projeto na formação dos acadêmicos e capacitação de residentes, os resultados descritos confirmam a importância das atividades de extensão universitária e de seu elo com o ensino e a pesquisa, corroborando ao estudo de Mascarenhas, da Silva e Torres (2021). Assim, nota-se que o projeto tem contribuído para a formação e capacitação dos futuros profissionais, alcançando os objetivos propostos e pautados na indissociabilidade da tríade ensino-extensão- pesquisa.

Diante disso, é importante que mais projetos de extensão como este, envolvendo acadêmicos, residentes, docentes, preceptores, tutores e a família, sejam fomentados, pois podem favorecer o desenvolvimento adequado do lactente, em especial, aquele considerado de risco, bem como, contribuir para a formação e capacitação dos futuros profissionais.

\section{CONSIDERAÇÕES FINAIS}

Considerando a população de lactentes, o projeto tem propiciado atendimento especializado, com detecção e intervenção precoce para lactentes considerados de risco da cidade e da região, permitindo estimular a aquisição das habilidades motoras nos dois primeiros anos de vida, contribuindo para um melhor desenvolvimento motor de lactentes.

A participação dos pais e/ou cuidadores durante o acompanhamento e intervenção, seguindo as orientações, favorece a percepção e o direcionamento do cuidado para o desenvolvimento do lactente, propiciando efeito positivo da intervenção precoce.

Do ponto de vista acadêmico e profissional, o projeto tem favorecido a inter-relação entre docentes, residentes, acadêmicos e família, permitindo incrementar a formação dos graduandos em fisioterapia e dos residentes na área de atenção à saúde da criança, pautado no atendimento do lactente em sua integralidade e com cuidado humanizado.

Extensio: R. Eletr. de Extensão, ISSN 1807-0221 Florianópolis, v. 18, n. 39, p. 25-35, 2021. 
Intervenção fisioterapêutica precoce em lactentes de risco: uma vivência baseada em evidências

\section{REFERÊNCIAS}

ALMEIDA, Bruna et al. Fatores de risco para o parto prematuro em uma maternidade estadual de referência. Saúde (Sta. Maria), v. 44, n. 2, p. 1-10, 2018.

CABRAL, Thais et al. Analysis of sensory processing in preterm infants. Early Human Development, v. 103, p. 77-81, 2016.

CHASKEL, Roberto et al. Alteraciones em el neurodesarrollo em pré-escolares com antecedente de prematurez: un estudio de corte. Rev.fac.med., v. 26, n. 1, p. 45-54, 2018.

FORMIGA, Cibelle K. M. R.; PEDRAZZANI, Elisete S.; TUDELLA, Eloisa. Intervenção precoce com bebês de risco. Rio de Janeiro: Atheneu, P. 131-160, 2010.

FORMIGA, Cibelle K. M. R.; RAMOS, Bruna A. Programas de intervenção precoce: Orientações gerais e experiências. Revista Dialogo e Perspectivas em educação especial, v. 3, n. 2, 2016.

FORMIGA, Cibelle Kayenne Martins Roberto; LINHARES, Maria Beatriz Martins. Avaliação do desenvolvimento inicial de crianças nascidas pré-termo. Revista da Escola de Enfermagem da USP, v. 43, p. 472-480, 2009.

GRANZOTTO, José A.; FONSECA, Silvia S.; LINDEMANN, Flavio L. Fatores relacionados com a mortalidade neonatal em uma Unidade de Terapia Intensiva neonatal na região Sul do Brasil. Revista da AMRIGS, v. 56, n. 1, p. 57-62, 2012.

GUIMARÃES, E. L. Estudo para detecção precoce de sinais indicativos de alterações no desenvolvimento neurosensóriomotor em bebês de risco. 159 f. Dissertação de mestrado, Universidade Federal de São Carlos, São Carlos, São Paulo, Brasil, 2001.

GUIMARÃES, E. L; TUDELLA, E. Estudo para detecção precoce de sinais indicativos de alterações no desenvolvimento neuro-sensório-motor em bebês de risco. São Carlos (SP): Universidade Federal de São Carlos, 2003.

KREY, Francieli et al. Alterações respiratórias relacionadas à prematuridade em terapia intensiva neonatal. Revista Rene, v.17, n.6, p. 766-773, 2016.

MARINI, Bruna P. R.; LOURENÇO, Mariane C.; BARBA, Patricia, C. S. D. Revisão sistemática integrativa da literatura sobre modelos e práticas de intervenção precoce no Brasil. Revista Paulista de Pediatria, v. 35, n. 4, p. 456-463, 2017.

MASCARENHAS, Anne Lizabelle Leite Duarte; DA SILVA, Márcia Regina Farias; TORRES, Maria Betânia Ribeiro. A posição da extensão universitária no espaço acadêmico brasileiro: aspectos legais e campo de lutas. Extensio: Revista Eletrônica de Extensão, v. 18, n. 38, p. 2-16, 2021. 
Intervenção fisioterapêutica precoce em lactentes de risco: uma vivência baseada em evidências

MESQUITA, Mirta; CASARTELLI, Marco. Hiperbilirrubinemia neonatal, encefalopatía bilirrubínica aguda y Kernicterus: La secuencia sigue vigente en el siglo XXI. Pediatr.

(Asunción), Asunción, v. 44, n. 2, p. 153-158, 2017.

NOVAK I, MORGAN C. High-risk follow-up: Early intervention and rehabilitation. Handb Clin Neurol, v. 162, p. 483-510, 2019.

NutriSUS. Portal da Secretaria de Atenção Primária a Saúde (SAPS), 2015. PIPER MC, DARRAH J. Motor assessment of the developing infant. EUA WB: Saunders Company; 1994.

SABOUTE, Maryam et al. The effect of intensive phototherapy on management of hyperbilirubinemia in neonates with the gestational age of 34 weeks and more. Iranian Journal of Neonatology IJN, Mashhad University of Medical Sciences, v. 8, n. 4, p. 83-88, 2017.

SANTOS, Daniel D.; PORTO, Julia A.; LERNER, Rogério. O Impacto do Desenvolvimento na Primeira Infância sobre a Aprendizagem. Comitê Científico Núcleo Ciência Pela Infância, 2014.

SANTOS, Heloisa C. V.; PACHECO, Márcia M. D. R. Os fatores de risco ao desenvolvimento da criança em contextos de creche pública. Revista Exitus, v. 6, n. 2, p. 48-67, 2016.

SAVIO, Josiani et al. Perfil clínico de neonatos internados em uma UTI do sul catarinense. Inova Saúde, v. 5, n. 1, p. 117-128, 2016.

SETUMBA, Mario et al. Mortalidade em recém-nascidos de baixo peso ao nascer: limites e desafios para o acesso universal. Port J Public Health, v. 36, n. 2, p. 95-101, 2018.

SILVA, Carla C.V. Atuação da fisioterapia através da estimulação precoce em bebes prematuros. Revista Eletrônica Atualiza Saúde, v. 5, n. 5, p. 29-36, 2017.

SILVA, Polyana et al. Influence of maternal age in perinatal conditions in live births of São Luís, Maranhão. Revista Fun Care Online, v.12, p. 281-287, 2020.

Urzêda, RN, et al. Reflexos, reações e tônus muscular de bebês pré-termo em um programa de intervenção precoce. Revista Neurociências, v. 17(4), p. 319-325, 2009.

VALVERDE, Bianca B.; JURDI, Andréa P. S. Análise das Relações entre Intervenção Precoce e Qualidade de Vida Familiar. Rev. bras. educ. espec., Bauru, v. 26, n. 2, p. 283-298, 2020.

WALKER, Susan et al. Child development: risk factors for adverse outcomes in developing countries. The Lancet, v. 369, n. 9556, p. 145-157, 2007.

Recebido em: 17/08/2020

Aceito em: 12/08/2021

Extensio: R. Eletr. de Extensão, ISSN 1807-0221 Florianópolis, v. 18, n. 39, p. 25-35, 2021. 